\title{
Real-world treatment duration in ALK-positive non-small-cell lung cancer patients receiving brigatinib through the early access program
}

\author{
Huamao M Lin*,1, Xiaoyun Pan², Peijie Hou ${ }^{3}$, Susan Allen ${ }^{1}$, Pia Baumann ${ }^{1}$ \& Maximilian J \\ Hochmair ${ }^{4}$ \\ ${ }^{1}$ Millennium Pharmaceuticals, Inc., Cambridge, MA 02139, USA \\ ${ }^{2}$ EMD Serono, Inc., Billerica, MA 01821, USA \\ ${ }^{3}$ Regeneron Pharmaceuticals, Inc., Tarrytown, NY 10591, USA \\ ${ }^{4}$ Karl Landsteiner Institute of Lung Research \& Pulmonary Oncology, Department of Respiratory \& Critical Care Medicine, \\ Krankenhaus Nord, Vienna, Austria \\ *Author for correspondence: Tel.: +1 617441 6930; Fax: +1 617932 5114; mark.lin@takeda.com
}

\begin{abstract}
Aim: To assess time-to-treatment discontinuation (TTD) of brigatinib following treatment with ALK tyrosine kinase inhibitor(s) (TKIs) in patients with ALK-positive (ALK+) non-small-cell lung cancer (NSCLC) receiving brigatinib through the international early access program. Patients $\&$ analysis: Analysis was performed for patients with ALK+ NSCLC treated with prior ALK TKIs, including next-generation ALK TKIs. Results: Data for 604 patients (21 countries), including patients with prior next-generation ALK TKIs, were reported. The median TTD of brigatinib in patients with prior crizotinib, alectinib, ceritinib or lorlatinib was 10.0, 8.7, 10.3 and 7.5 months, respectively. Conclusion: Brigatinib appears to be effective and tolerable in real-world clinical practice regardless of prior treatment with first or NG ALK TKIs.
\end{abstract}

First draft submitted: 23 December 2019; Accepted for publication: 20 March 2020; Published online: 27 April 2020

Keywords: alectinib • anaplastic lymphoma kinase positive non-small-cell lung cancer $\bullet$ brigatinib $\bullet$ ceritinib $\bullet$ compassionate use $\bullet$ crizotinib $\bullet$ early access $\bullet$ lorlatinib $\bullet$ real-world evidence $\bullet$ treatment duration

Lung cancer is the fourth most common type of cancer worldwide, with an estimated 2.1 million new cases diagnosed and 1.8 million deaths each year [1]. Non-small-cell lung cancer (NSCLC) represents $85 \%$ of all lung cancer cases, and the majority of patients present with advanced disease [2]. Genomic analyses indicate that $A L K$ gene rearrangements are responsible for 3-5\% of NSCLC cases, mainly of the adenocarcinoma histotype [3-5]. While lung cancer often affects individuals of older age with a smoking history, ALK-positive (ALK+) NSCLC patients are usually younger (median age of 51 years) and light or nonsmokers [6].

Until recently, the first-line (1L) treatment option for patients with ALK+ NSCLC was the first-generation ALK tyrosine kinase inhibitor (TKI) crizotinib [7-9]. However, most patients develop resistance to crizotinib due to multiple resistance mechanisms, including secondary mutations, and experience progression within 12 months [10]. The brain is a sanctuary metastatic site in ALK+ NSCLC, and due to poor central nervous system (CNS) coverage of crizotinib, the majority of crizotinib-treated patients develop brain metastases $[10,11]$. Numerous nextgeneration (NG) ALK TKIs have been developed, including ceritinib, alectinib, brigatinib and lorlatinib, allowing the possibility of sequencing these agents to extend patient benefit and improve outcomes [12-18]. These NG ALK TKIs have a wide spectrum of activity against crizotinib-resistant $A L K$ mutations, and are more potent and have better CNS penetration than crizotinib $[19,20]$.

The current European Society for Medical Oncology (ESMO) and National Comprehensive Cancer Network $(\mathrm{NCCN})$ treatment guidelines for ALK+ NSCLC recommend alectinib, crizotinib, ceritinib or brigatinib in the 1L setting, although brigatinib has not received European Medicines Agency (EMA) or US FDA approval as a $1 \mathrm{~L}$ therapy $[8,9]$. For patients with oligometastatic progression, local treatment, such as surgery or radiotherapy,

Future Medicine 
and continued targeted treatment with ALK TKIs are recommended [8,9]. Patients with systemic progression on crizotinib, particularly with CNS involvement, should receive alectinib, ceritinib or brigatinib; however, the optimal targeted treatment has not been established $[8,9,19,20]$. NCCN also recommends systemic therapy options such as PD-1/PD-L1 inhibitor monotherapy for patients with multiple lesions [11]. ESMO guidelines recommend lorlatinib only to patients progressing on an NG ALK TKI [8]. In the USA, the FDA granted accelerated approval of lorlatinib, based on tumor response rate and duration of response, for patients with ALK+ metastatic NSCLC whose disease has progressed on crizotinib and $\geq 1$ other ALK inhibitor for metastatic disease; or whose disease has progressed on alectinib or ceritinib as the first ALK inhibitor therapy for metastatic disease [21].

Platinum-based chemotherapy and the four-drug regimen (atezolizumab plus bevacizumab, paclitaxel and carboplatin; not EMA approved) are the only ESMO recommendations for patients with metastatic disease who fail targeted therapy with ALK TKIs [8]. The IMpower150 randomized, open-label Phase III study aimed to test the efficacy of the four-drug regimen against the standard-of-care bevacizumab plus carboplatin and paclitaxel in chemotherapy-naive patients with metastatic NSCLC (mNSCLC) [22]. This study included a limited number of patients with ALK+ NSCLC $(n=40)$, and the results were reported as part of the larger subgroup of patients with EGFR-mutation-positive NSCLC (EGFR + status $n=124)$; thus, the benefits of the four-drug regimen in ALK+ NSCLC patients are unclear [22]. The NCCN recommends continued ALK TKI treatment and local therapy for patients progressing on targeted therapies, and advises lorlatinib or systemic therapy options for those progressing on NG ALK TKI with multiple systemic lesions [9].

The international brigatinib early access program (EAP) was initiated in July 2016 to enable early access to brigatinib in response to an unsolicited request for patients with ALK+ NSCLC who received prior ALKTKIs and were unable to participate in clinical studies. At the time of the analysis, more than 20 countries participated in the program. The primary objective of the brigatinib EAP was to enable access for eligible patients with an unmet need; any data generated are incidental. Nevertheless, accumulating evidence supports the benefit of brigatinib in patients with ALK+ NSCLC in the real-world setting from individual countries [23,24].

The objective of this retrospective observational study of data from the EAP was to assess real-world treatment duration of brigatinib following treatment with ALK TKIs in patients with ALK+ NSCLC who received treatment through the international brigatinib EAP.

\section{Materials \& methods}

Participants

Adult ( $\geq 18$ years) patients were eligible for treatment with brigatinib in the EAP if they: had histologically or cytologically confirmed locally advanced NSCLC (aNSCLC) or mNSCLC; had an ALK rearrangement detected according to local standard procedure; received prior ALK TKI (before June 2018) or were resistant to or intolerant of crizotinib (after June 2018); and could not be adequately treated with medications approved or available through clinical trials in their country of residence. At the time of the analysis, this international EAP included 604 patients in multiple countries from the Americas, Asia and Europe [25].

\section{Outcomes}

Patient baseline demographics \& clinical characteristics

Patient data including age, gender, latest performance status (PS) and therapies received prior to treatment with brigatinib were collected as part of the initial eligibility screening to receive access to brigatinib through the EAP. The data were entered into a database with a cut-off date of November 2018.

\section{Brigatinib time-to-treatment discontinuation}

Time-to-treatment discontinuation (TTD) is an end point calculated using real-world data, and is defined as the time from the start of a therapy to the time when treatment is discontinued for any reason. Brigatinib TTD was selected as an efficacy end point, as it was consistently captured through voluntary, physician-reported data on discontinuation forms. Discontinuation was either confirmed for patients with a discontinuation form or assumed if there was a gap of $>120$ days between data cut-off date (November 2018) and last medication shipment date. Patients who did not meet these criteria were censored at the end of available follow-up. TTD was estimated from Kaplan-Meier curves. Reasons for brigatinib discontinuation were summarized as frequency counts and percentages. 


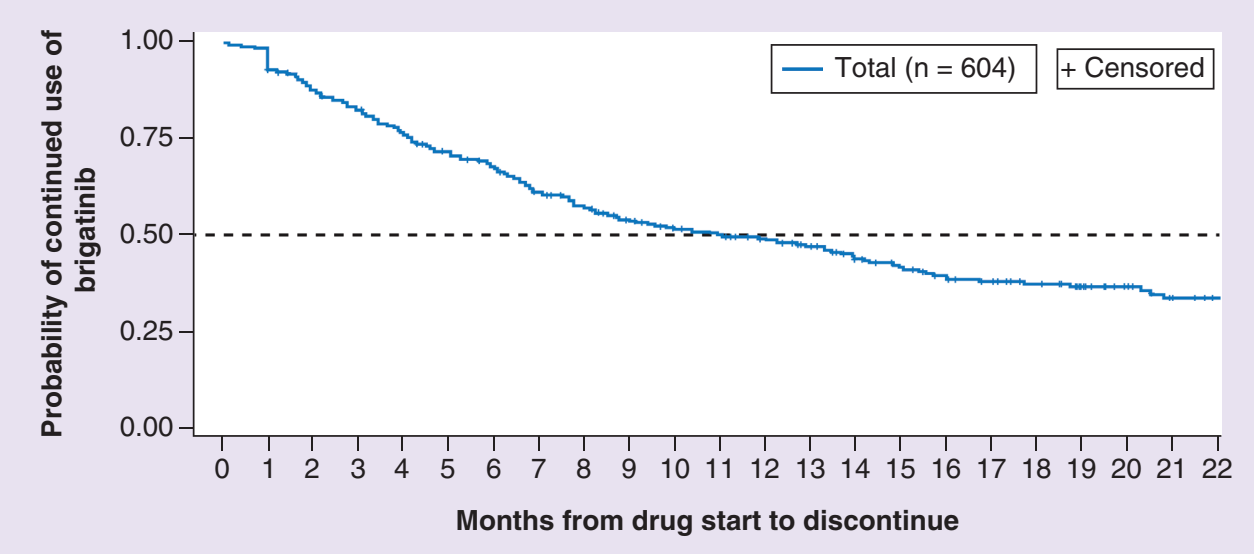

\begin{tabular}{|l|c|c|c|c|c|c|c|c|c|}
\hline Month & 1 & 2 & 3 & 6 & 9 & 12 & 15 & 18 & 21 \\
\hline $\begin{array}{l}\text { 0+ probability of } \\
\text { continued } \\
\text { use of brigatinib (\%) }\end{array}$ & 92.7 & 87.5 & 82.6 & 67.1 & 53.4 & 48.6 & 41.4 & 37.2 & 33.7 \\
\hline
\end{tabular}

Figure 1. Probability of continued use of brigatinib across all lines of therapy.

\section{Statistical analyses}

Data were analyzed using SAS software (version 9.4). Brigatinib TTD and probability of continued use of brigatinib were estimated from Kaplan-Meier curves. Subgroup analyses were conducted based on whether patients had received crizotinib, alectinib, ceritinib or lorlatinib prior to brigatinib and by number of ALK inhibitors received prior to brigatinib.

\section{Results}

\section{Participants \& baseline characteristics}

At the time of analysis, a total of 604 patients had received brigatinib through the EAP in countries including Argentina, Austria, Brazil, Estonia, Finland, France, Germany, Hong Kong, Italy, Mexico, The Netherlands, Norway, Portugal, Russian Federation, Singapore, Spain, Sweden, Switzerland, Taiwan, Turkey and the UK. The number of participants in each country was not available. Median patient age was 58.0 years, $56.8 \%$ of patients were females and $52.0 \%$ of patients had a PS of one (Table 1). Among patients with ALK TKI history prior to brigatinib, $64.2 \%$ had received two or fewer (median of two) ALK TKIs prior to brigatinib. Most patients (29.5\%) had received ceritinib as the most recent ALK TKI prior to brigatinib, 19.0\% of patients had received crizotinib as the most recent ALK TKI prior to brigatinib and 13.1\% had been treated with alectinib as the most recent ALK TKI (Table 1).

\section{Brigatinib TTD}

Across all lines of therapy

During the 27.5-month period over which the retrospective data analysis was conducted, median brigatinib TTD was 11.0 months (95\% CI: 8.7-13.9), and the probability of continued use of brigatinib was $67.1 \%$ at 6 months and $48.6 \%$ at 12 months (Figure 1 ).

\section{Subgroup analyses based on prior ALK TKIs}

Duration of brigatinib treatment was analyzed for subgroups of patients treated with various ALK TKIs prior to brigatinib. 
Table 1. Baseline characteristics of patients in the brigatinib early access program.

\section{Characteristics}

Age, median (range)

Gender

Males

Females

Missing

Latest performance status

0

1

2

Missing

Number of prior ALK TKIs

1

2

$3+$

Missing

Prior ALK TKI

Alectinib

Alectinib + Ceritinib

Alectinib + Ensartinib

Ceritinib

Ceritinib + Lorlatinib

Crizotinib

Crizotinib + Alectinib

Crizotinib + Alectinib + Ceritinib

Crizotinib + Alectinib + Ceritinib + Lorlatinib

Crizotinib + Alectinib + Lorlatinib

Crizotinib + Ceritinib

Crizotinib + Ceritinib + Lorlatinib

Crizotinib + Ensartinib

Crizotinib + Lorlatinib

Ensartinib

Entrectinib

Missing

Most recent ALK TKI

Alectinib

Ceritinib

Crizotinib

Crizotinib + Ceritinib

Ensartinib

Entrectinib

Lorlatinib

Missing

Data represented as $n(\%)$, unless otherwise stated.

EAP: Early access program; TKI: Tyrosine kinase inhibitor.
Patients $(n=604)$

$58.0(19-94)$

$256(42.4)$

$343(56.8)$

$5(0.8)$

205 (33.9)

$314(52.0)$

49 (8.1)

$36(6.0)$

155 (25.7)

209 (34.6)

69 (11.5)

171 (28.3)

8 (1.3)

$8(1.3)$

$1(0.2)$

28 (4.6)

$2(0.3)$

117 (19.4)

43 (7.1)

38 (6.3)

10 (1.7)

8 (1.3)

155 (25.7)

13 (2.2)

$1(0.2)$

$4(0.7)$

$1(0.2)$

$1(0.2)$

171 (28.3)

79 (13.1)

178 (29.5)

115 (19.0)

$1(0.2)$

$3(0.5)$

$1(0.2)$

31 (5.1)

196 (32.5)

Prior alectinib

Overall, 111 patients received alectinib as any line of therapy prior to brigatinib. These patients had a median TTD of brigatinib of 8.7 months (95\% CI: 7.5-14.9), and a probability of continued use of brigatinib of 71.5 and $47.4 \%$ at 6 and 12 months, respectively (Figure 2A). Among the 111 patients receiving alectinib, 79 had received alectinib as the most recent ALK TKI prior to brigatinib. These patients had a median TTD of brigatinib 

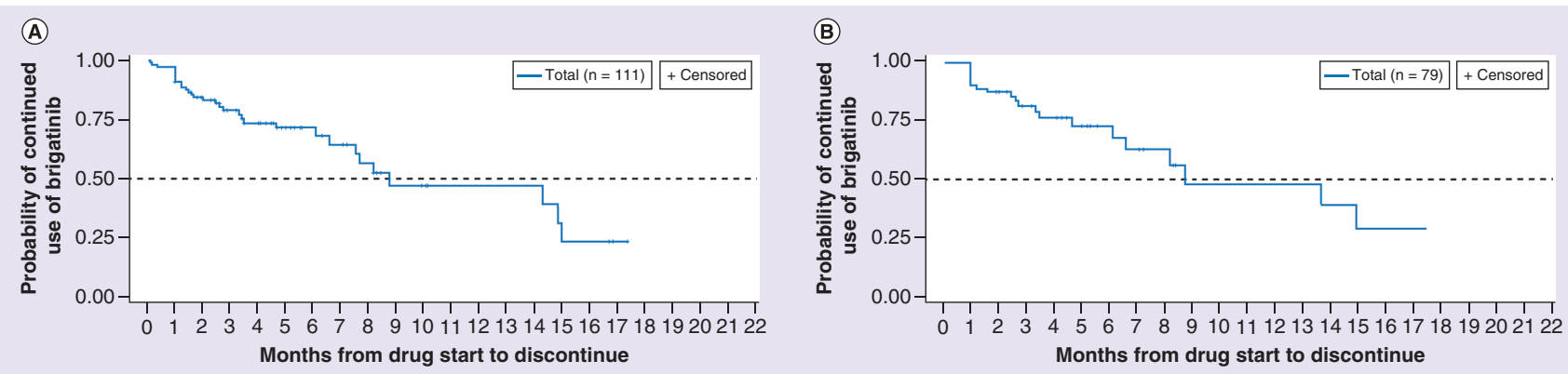

\begin{tabular}{|l|c|c|c|c|c|c|c|}
\hline Month & 1 & 2 & 3 & 6 & 9 & 12 & 15 \\
\hline $\begin{array}{l}\text { Probability of continued } \\
\text { use of brigatinib (\%) }\end{array}$ & 91.0 & 84.4 & 78.8 & 71.5 & 47.4 & 47.4 & 23.7 \\
\hline
\end{tabular}

(C)

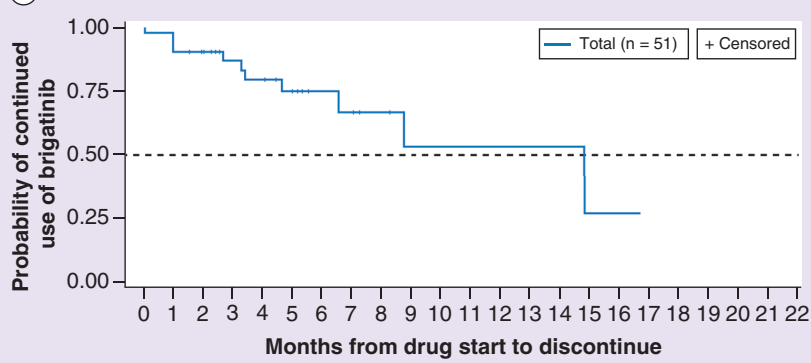

\begin{tabular}{|l|c|c|c|c|c|c|c|}
\hline Month & 1 & 2 & 3 & 6 & 9 & 12 & 15 \\
\hline $\begin{array}{l}\text { Probability of continued } \\
\text { use of brigatinib (\%) }\end{array}$ & 90.2 & 90.2 & 87.0 & 75.3 & 53.5 & 53.5 & 26.8 \\
\hline
\end{tabular}

\begin{tabular}{|l|c|c|c|c|c|c|c|}
\hline Month & 1 & 2 & 3 & 6 & 9 & 12 & 15 \\
\hline $\begin{array}{l}\text { Probability of continued } \\
\text { use of brigatinib (\%) }\end{array}$ & 89.9 & 86.8 & 80.7 & 72.6 & 48.2 & 48.2 & 28.9 \\
\hline
\end{tabular}

(D)

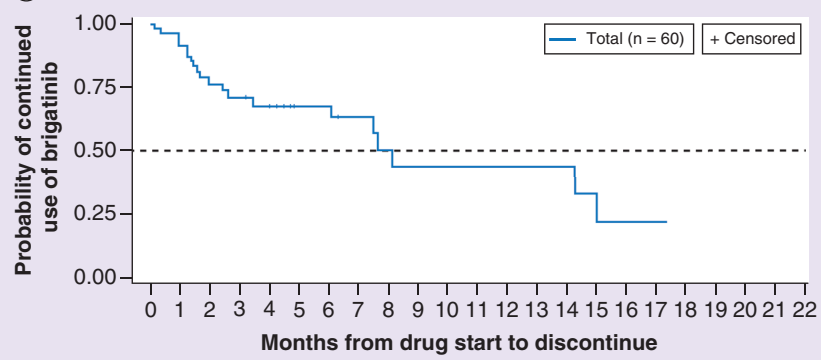

\begin{tabular}{|l|c|c|c|c|c|c|c|}
\hline Month & 1 & 2 & 3 & 6 & 9 & 12 & 15 \\
\hline $\begin{array}{l}\text { Probability of continued } \\
\text { use of brigatinib (\%) }\end{array}$ & 91.7 & 78.8 & 71.3 & 68.0 & 44.2 & 44.2 & 22.1 \\
\hline
\end{tabular}

(E) Median brigatinib TTD among patients with prior alectinib

\begin{tabular}{|l|l|l|}
\hline Prior TKI & $\mathbf{n}$ & Median brigatinib TTD $\mathbf{( 9 5 \% ~ C l )}$ \\
\hline Prior alectinib as any line & 111 & 8.7 months (7.5-14.9) \\
\hline Alectinib as most recent ALK TKI & 79 & 8.7 months (6.6-NE) \\
\hline $\begin{array}{l}\text { Crizotinib + alectinib or alectinib } \\
\text { alone }\end{array}$ & 51 & 14.8 months (6.6-NE) \\
\hline $\begin{array}{l}\geq 1 \text { other NG ALK TKI in addition } \\
\text { to alectinib }\end{array}$ & 60 & 8.1 months (6.1-14.9) \\
\hline
\end{tabular}

Figure 2. Brigatinib use among patients with prior alectinib. (A) Patients with prior alectinib as any line. (B) Patients with alectinib as most recent ALK TKI. (C) Patients with crizotinib + alectinib or alectinib alone. (D) Patients with $\geq 1$ other NG ALK TKI in addition to alectinib. (E) Median brigatinib TTD among patients with prior alectinib. NE: Not evaluable; NG: Next-generation; TKI: Tyrosine kinase inhibitor; TTD: Time-to-treatment discontinuation.

of 8.7 months (95\% CI: 6.6-not evaluable [NE]), and a 72.6 and $48.2 \%$ probability of continued use of brigatinib at 6 and 12 months, respectively (Figure 2B). Patients who had received crizotinib plus alectinib or alectinib alone $(\mathrm{n}=51)$ prior to brigatinib had a median TTD of brigatinib of 14.8 months (95\% CI: 6.6-NE), and a 75.3 and $53.5 \%$ probability of continued use of brigatinib at 6 and 12 months, respectively (Figure 2C). Patients who had previously received $\geq 1$ other NG ALK inhibitor (e.g., ceritinib or lorlatinib) in addition to alectinib $(\mathrm{n}=60)$ had a median TTD of 8.1 months (95\% CI: 6.1-14.9), and a 68.0 and $44.2 \%$ probability of continued use of brigatinib at 6 and 12 months, respectively (Figure 2D). The majority of these patients also received prior crizotinib. The median TTD of these subgroups is summarized in Figure 2E. 


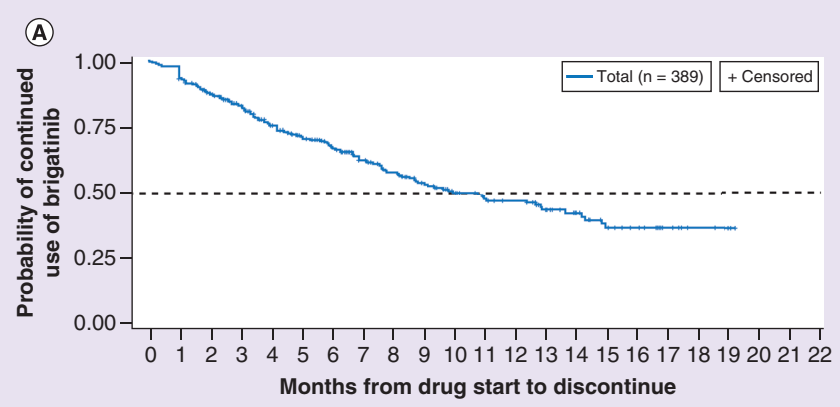

\begin{tabular}{|l|c|c|c|c|c|c|c|c|}
\hline Month & 1 & 2 & 3 & 6 & 9 & 12 & 15 & 18 \\
\hline $\begin{array}{l}\text { Probability of continued } \\
\text { use of brigatinib (\%) }\end{array}$ & 91.0 & 84.4 & 78.8 & 71.5 & 47.4 & 47.4 & 23.7 & 36.1 \\
\hline
\end{tabular}

(c)

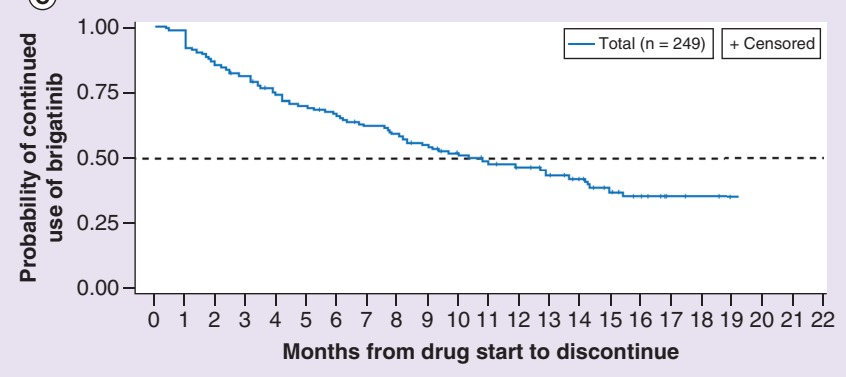

\begin{tabular}{|l|c|c|c|c|c|c|c|c|}
\hline Month & 1 & 2 & 3 & 6 & 9 & 12 & 15 & 18 \\
\hline $\begin{array}{l}\text { Probability of continued } \\
\text { use of brigatinib (\%) }\end{array}$ & 92.0 & 85.5 & 81.1 & 66.0 & 54.0 & 46.0 & 36.7 & 34.8 \\
\hline
\end{tabular}

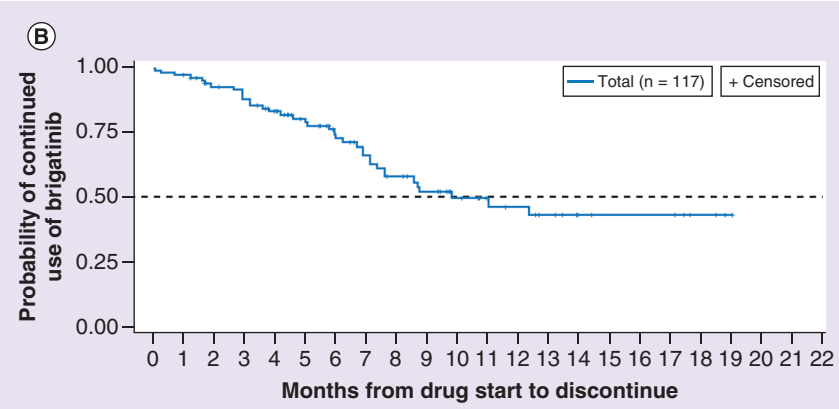

\begin{tabular}{|l|c|c|c|c|c|c|c|c|}
\hline Month & 1 & 2 & 3 & 6 & 9 & 12 & 15 & 18 \\
\hline $\begin{array}{l}\text { Probability of continued } \\
\text { use of brigatinib (\%) }\end{array}$ & 97.4 & 92.9 & 88.1 & 72.9 & 52.3 & 46.7 & 43.3 & 43.3 \\
\hline
\end{tabular}

(D)

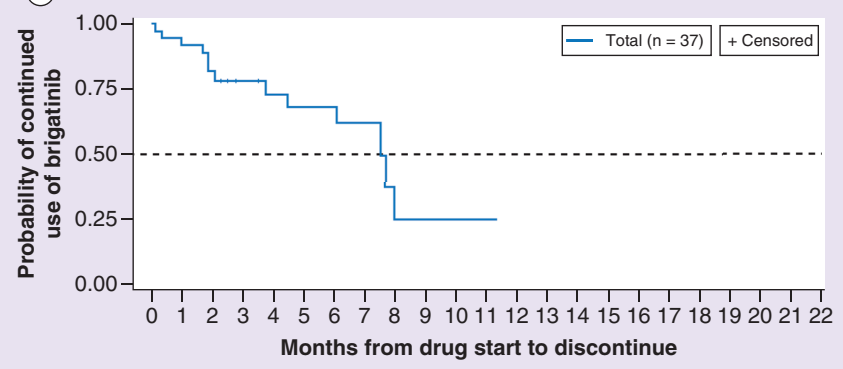

\begin{tabular}{|l|c|c|c|c|c|}
\hline Month & 1 & 2 & 3 & 6 & 9 \\
\hline $\begin{array}{l}\text { Probability of continued } \\
\text { use of brigatinib (\%) }\end{array}$ & 91.9 & 81.8 & 78.1 & 68.3 & 24.8 \\
\hline
\end{tabular}

(E) Median brigatinib TTD among patients with prior crizotinib or lorlatinib

\begin{tabular}{|l|l|l|}
\hline Prior TKI & $\mathbf{n}$ & Median brigatinib TTD $(\mathbf{9 5} \% \mathbf{C I})$ \\
\hline Prior crizotinib as any line & 389 & 10.0 months $(8.2-13.6)$ \\
\hline Prior crizotinib alone & 117 & 9.8 months $(7.3-N E)$ \\
\hline Prior ceritinib as any line & 249 & 10.3 months $(8.1-13.6)$ \\
\hline Prior lorlatinib as any line & 37 & 7.5 months $(4.5-N E)$ \\
\hline
\end{tabular}

Figure 3. Brigatinib use among patients with prior ALK tyrosine kinase inhibitors. (A) Patients with prior crizotinib as any line. (B) Patients with prior crizotinib alone. (C) Patients with prior ceritinib as any line. (D) Patients with prior lorlatinib as any line. (E) Median brigatinib TTD among patients with prior crizotinib or lorlatinib.

NE: Not evaluable; TKI: Tyrosine kinase inhibitor; TTD: Time-to-treatment discontinuation.

Prior crizotinib, ceritinib or lorlatinib

Patients who had received crizotinib as any line of therapy prior to brigatinib $(\mathrm{n}=389)$ had a median TTD of brigatinib of 10.0 months (95\% CI: 8.2-13.6), and a 66.9 and $46.6 \%$ probability of continued use of brigatinib at 6 months and 12 months, respectively (Figure 3A). Those who received crizotinib with no other prior ALK TKI before initiating brigatinib $(\mathrm{n}=117)$ had a median TTD of brigatinib of 9.8 months (95\% CI: 7.3-NE), and a 72.9 and $46.7 \%$ probability of continued use of brigatinib at 6 and 12 months, respectively (Figure 3B). Patients receiving ceritinib treatment as any line of therapy prior to brigatinib $(n=249)$ had a median TTD of brigatinib of 10.3 months (95\% CI: 8.1-13.6), and a 66.0 and $46.3 \%$ probability of continued use of brigatinib at 6 and 12 months, respectively (Figure 3C). Patients who had received lorlatinib treatment as any line of therapy prior to brigatinib $(\mathrm{n}=37)$ had a median TTD of brigatinib of 7.5 months $(95 \% \mathrm{CI}: 4.5-\mathrm{NE})$, and a $68.3 \%$ probability 


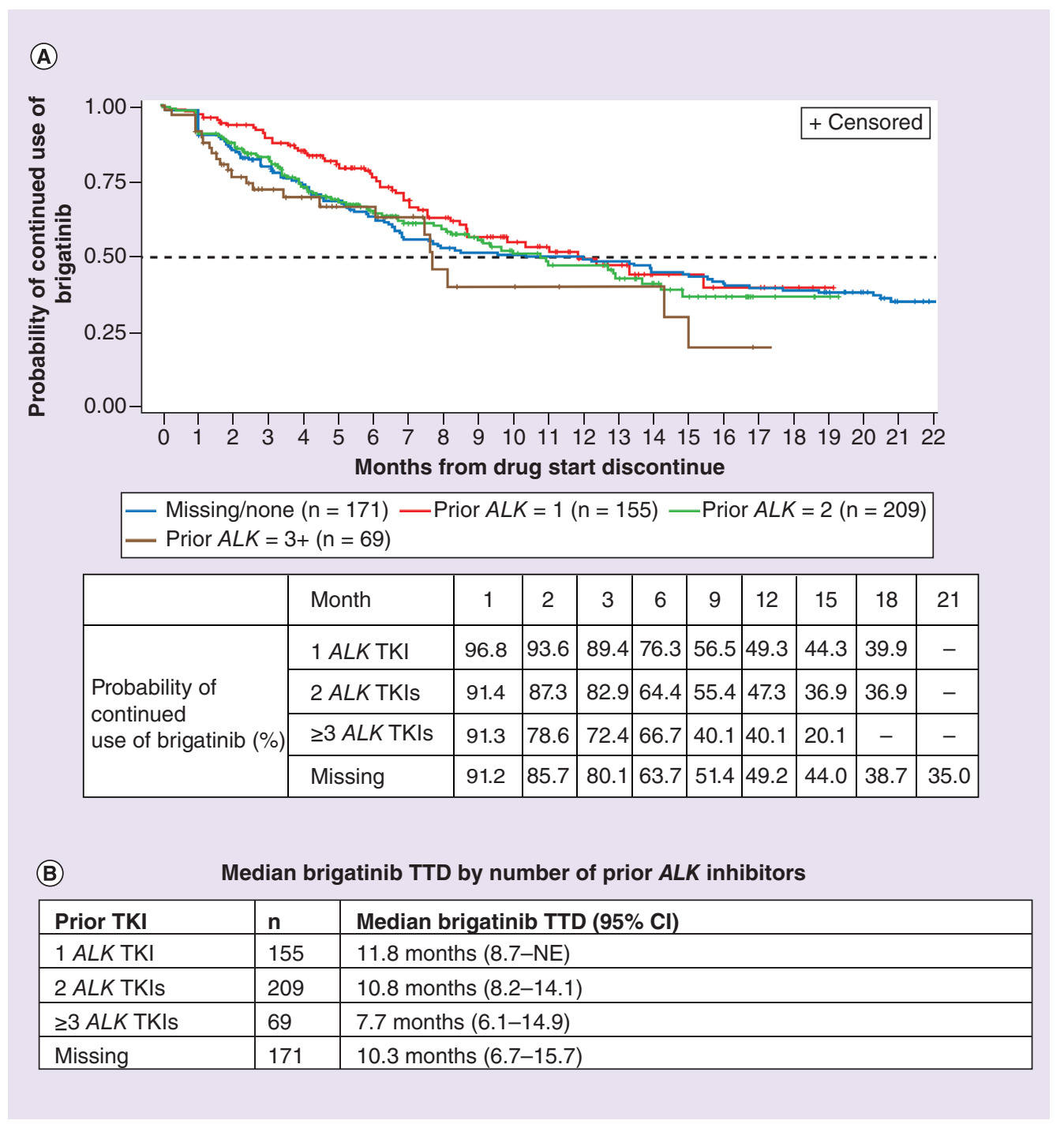

Figure 4. Brigatinib use by number of prior ALK tyrosine kinase inhibitors. (A) Patients with one to three prior ALK TKIs. (B) Median brigatinib TTD by number of prior ALK inhibitors.

NE: Not evaluable; TKI: Tyrosine kinase inhibitor; TTD: Time-to-treatment discontinuation.

of continued use of brigatinib at 6 months (Figure 3D). The median TTD of these subgroups are summarized in Figure 3E.

Number of prior ALK TKIs

Patients were also grouped by number of ALK TKIs prior to brigatinib. Out of the 155 receiving one prior ALK TKI, 117 received crizotinib (Table 1). Patients with one ALK TKI prior to brigatinib had a median TTD of brigatinib of 11.8 months ( $95 \%$ CI: 8.7-NE), and a 76.3 and $49.3 \%$ probability of continued use of brigatinib at 6 and 12 months, respectively. Patients receiving two ALK TKIs prior to brigatinib $(\mathrm{n}=209)$ had a median TTD of brigatinib of 10.8 months (95\% CI: 8.2-14.1), and a 64.4 and $47.3 \%$ probability of continued use of brigatinib at 6 and 12 months, respectively. Patients receiving three or more ALK TKIs prior to brigatinib $(\mathrm{n}=69)$ had a median TTD of brigatinib of 7.7 months (95\% CI: 6.1-14.9), and a 66.7 and $40.1 \%$ probability of continued use of brigatinib at 6 and 12 months, respectively. Among patients with missing information on the number of ALK TKIs prior to brigatinib $(n=171)$, the median TTD of brigatinib was 10.3 months (95\% CI: 6.7-15.7), and the probability of continued use of brigatinib was $63.7 \%$ at 6 months and $49.2 \%$ at 12 months (Figure 4). 
Table 2. Reasons for brigatinib treatment discontinuation.

\begin{tabular}{|l|l|}
\hline Reason for treatment discontinuation & $n(\%)$ \\
\hline Total patients & 604 \\
\hline Discontinued & $260(43.0)$ \\
\hline Adverse event & $4(0.7)$ \\
\hline Disease progression & $64(10.6)$ \\
\hline Death & $15(2.5)$ \\
\hline Lost to follow-up & $1(0.2)$ \\
\hline Other & $31(5.1)$ \\
\hline Assumed discontinued (discontinuation defined by gap) ${ }^{\dagger}$ & $145(24.0)$ \\
\hline $\begin{array}{l}\dagger \\
\text { Discontinuation was assumed for patients without confirmed discontinuation if there was a gap of }>120 \text { days between data cutoff date (7 November }\end{array}$
\end{tabular}

\section{Reasons for discontinuation}

Out of the 604 patients enrolled in the EAP, 260 (43.0\%) had discontinued brigatinib at the time of the analysis. Few patients reported discontinuation due to adverse events $(n=4,0.7 \%), 64(10.6 \%)$ had disease progression, and $15(2.5 \%$ ) patients died (Table 2). $24 \%$ of patients were assumed discontinued but did not indicate a reason for ending treatment.

\section{Discussion}

Since its inception, the international brigatinib EAP has enabled patients with ALK+ NSCLC from more than 20 countries to access brigatinib, an NG ALK TKI, providing real-world evidence (RWE) of brigatinib safety and efficacy in clinical practice. Patients were granted access to brigatinib as long as there was a clinical benefit, and TTD was used as a proxy end point to assess the efficacy of brigatinib. A recent post hoc analysis of 18 randomized clinical trials involving 8947 patients supports the use of TTD as a potential end point to assess the efficacy of therapies for mNSCLC. The post hoc analysis assessed the relationship between TTD, progression-free survival (PFS), and overall survival (OS) in trials that were submitted to the FDA as part of New Drug Applications or Biologic License Applications [26]. Findings showed TTD was more closely associated with PFS ( $\mathrm{r}=0.87$; 95\% CI: 0.86-0.87) than with OS ( $\mathrm{r}=0.68$; 95\% CI: 0.67-0.69) across therapeutic classes [26]. The median TTD exceeded median PFS, particularly in the oncogene-directed targeted therapy subgroup, which included EGFR and ALK. This may reflect the common practice of continued treatment beyond objective disease progression as long as a measurable benefit can be extended to the patient, as defined by response evaluation criteria in solid tumors [26].

For patients included in the brigatinib EAP, the median TTD of brigatinib was 11.0 months, despite a heterogeneous patient population pretreated with multiple ALK inhibitors. Continuous use of brigatinib was seen post-alectinib with a median TTD of 8.7 months, and post-ceritinib with a median TTD of 10.3 months. Brigatinib was also used post-lorlatinib, with a median TTD of 7.5 months. Consistent with previous real-world data for patients treated with NG ALK TKIs, brigatinib was well tolerated, with few observed adverse events [15-17,27-29].

An ongoing Phase II trial to investigate the activity of brigatinib in patients whose disease has progressed on prior NG ALK TKIs (ClinicalTrials.gov identifier: NCT02706626) will further refine the feasibility of a sequencing strategy for brigatinib use in this patient population [30]. Preliminary results from this Phase II trial showed a median PFS (mPFS) of 6.4 months (95\% CI: 4.6-NE) at a median follow-up of 6.7 months. The patients in this study were pretreated with a median of three prior ALK TKIs, which may account for the relatively shorter PFS as compared with the brigatinib TTD of 11.0 months (95\% CI: 8.7-13.9) reported in the EAP across all lines of therapy [30]. The results from the Phase II trial suggest brigatinib has activity after progression on NG ALK TKI in patients with stage IIIB/IV ALK+ NSCLC [30]. An additional study is underway to further address the efficacy of brigatinib after progression on alectinib or ceritinib (ClincialTrials.gov identifier: NCT03535740).

Previous reports have demonstrated real-world effectiveness of brigatinib. The BRIGALK study was a retrospective analysis of 104 patients with ALK+ NSCLC admitted into the brigatinib EAP in France from 1 September 2016 to 1 January 2018 [23]. Among patients pretreated with $\geq 2$ ALK TKIs, mPFS was 6.6 months (95\% CI: 4.8-9.9) with a median OS of 17.2 months (95\% CI: $11.0-$ not reached) [23]. At brigatinib initiation, these patients had a poorer PS (PS 0-1: 59.1\%, PS $\geq 2$ : 40.9\%) than those in the present study (PS 0-1: 85.9\%, PS 2: 8.1\%) [23]. In an independent report of patients with ALK+ NSCLC receiving brigatinib at a single institution 
in Austria, the overall mPFS was 9.9 months, whereas the largest treatment cohort (patients receiving brigatinib after crizotinib failure) showed an mPFS of 8.4 months [24]. In another real-world data collection in the broader brigatinib EAP conducted in Europe (UVEA-Brig), which included data from Italy, Norway, Spain and the UK, mPFS was 5.7 months [31]. The observed differences in the effectiveness of brigatinib in the real-world setting could reflect dissimilar patient characteristics, varied treatment history, disparities in regional care paradigms and measured clinical outcomes. Taken together, these data suggest that brigatinib is effective in real-world clinical practice regardless of previous treatment with NG ALK TKIs.

Treatment of ALK+ NSCLC has rapidly evolved from crizotinib as the standard 1L therapy in 2015 to NG ALK TKIs as current initial therapy. As patients relapse on NG ALK TKIs, optimal sequencing of treatment is currently being investigated. The results of this study indicate that brigatinib may be associated with a clinical benefit in the real-world setting, as patients remained on therapy for a considerable duration after NG ALK TKIs, including alectinib.

\section{Limitations}

This study was associated with several limitations. First, data analyzed from the EAP were collected from access forms, shipment records and voluntary discontinuation forms, and may be subject to error. Second, quantification of therapeutic efficacy, particularly when it is dependent on line of treatment, should be interpreted with caution. TTD is not an established end point, and it is contingent upon a patient's clinical circumstance and their assessment by physicians. Patients and physicians may be inclined to discontinue treatment early to receive a newer agent, shortening the TTD, and if fewer alternate treatment options exist, patients and physicians may persist on treatment longer, lengthening the TTD. Current treatment guidelines for oligometastatic progression recommend continued targeted treatment with the addition of local therapy, which may also lengthen the TTD. Further research is needed to validate TTD as an end point for use in pragmatic real-world trials. Third, the limited population of terminally-ill, heavily pre-treated patients with aNSCLC and short follow-up may have influenced the observed TTD. Fourth, AEs were collected in a database but not linked to discontinuation, and detailed AE analysis is still ongoing. Fifth, data on chemotherapy regimens were not captured. Last, lack of randomization in RWE studies is a source of variability, introducing potential for bias from unknown confounding variables obscuring treatment effects.

\section{Conclusion}

This retrospective observational study of data from the EAP in over 600 patients with ALK+ NSCLC pretreated with ALK TKI provides RWE of the efficacy and safety of brigatinib. The median TTD of brigatinib observed among patient subpopulations treated with prior alectinib, ceritinib or lorlatinib are encouraging and suggest brigatinib can provide clinical benefit for patients with progressive disease regardless of prior line of therapy. The safety profile of brigatinib was largely consistent with previous reports.

\section{Author contributions}

All authors contributed to the study design, data analysis and manuscript development.

\section{Financial \& competing interests disclosure}

This study was funded by Millennium Pharmaceuticals, Inc., a wholly owned subsidiary of Takeda Pharmaceutical Company Limited. HM Lin, S Allen and P Baumann are employees of Millennium Pharmaceuticals, Inc., a wholly owned subsidiary of Takeda Pharmaceutical Company Limited, and may own stock. At the time of study, X Pan and P Hou were employees of Millennium Pharmaceuticals, Inc., a wholly owned subsidiary of Takeda Pharmaceutical Company Limited, and may own stock. MJ Hochmair has received honoraria from AstraZeneca, Bristol-Myers Squibb, Boehringer Ingelheim, Merck Sharp \& Dohme, Pfizer and Roche, and had consulting or advisory roles with Boehringer Ingelheim, Merck Sharp \& Dohme, Novartis, and Roche. Medical writing and editorial assistance funded by Millennium Pharmaceuticals, Inc., a wholly owned subsidiary of Takeda Pharmaceutical Company Limited. The authors have no other relevant affiliations or financial involvement with any organization or entity with a financial interest in or financial conflict with the subject matter or materials discussed in the manuscript apart from those disclosed.

The authors wish to acknowledge medical writing and editorial assistance provided by J Dembowy and J Kondejewski of SNELL Medical Communication Inc. 


\section{Summary points}

- The brigatinib international early access program (EAP) commenced in 2016 to enable unsolicited expanded access to brigatinib for patients with ALK + non-small-cell lung cancer (NSCLC) who had no other effective therapeutic options and were unable to participate in a clinical study.

- Real-world data on brigatinib in patients with ALK+ NSCLC previously treated with ALK tyrosine kinase inhibitors (TKIs), including next-generation ALK TKIs alectinib, ceritinib or lorlatinib, could be extracted from the EAP.

- As of November 2018, data for 604 patients in 21 countries enrolled in the EAP could be collected.

- Time-to-treatment discontinuation (TTD) was used as a proxy for tolerability and effectiveness of brigatinib in the absence of reported clinical outcomes in the EAP.

- Brigatinib TTD in patients with previous crizotinib, alectinib, lorlatinib or ceritinib was 10.0, 8.7, 7.5 and 10.3 months, respectively.

- Among patients who received one previous ALK TKI, brigatinib TTD was 11.8 months, while in those receiving two or three prior ALK TKIs, brigatinib TTD was 10.8 and 7.7 months, respectively.

- Based on voluntary physician-reported data on discontinuation forms, the majority of patients $(57.0 \%)$ remained in the EAP and few had discontinued brigatinib treatment due to adverse events $(0.7 \%)$ or disease progression (10.6\%). $2.5 \%$ of patients died while participating in the EAP and $24 \%$ of patients were assumed discontinued but did not indicate a reason for discontinuation.

- The encouraging efficacy of brigatinib observed in the EAP suggests that brigatinib is effective and tolerable in the real-world setting regardless of previous ALK TKI treatment.

\section{Open access}

This work is licensed under the Attribution-NonCommercial-NoDerivatives 4.0 Unported License. To view a copy of this license, visit http://creativecommons.org/licenses/by-nc-nd/4.0/

\section{References}

Papers of special note have been highlighted as: $\bullet$ of interest

1. Bray F, Ferlay J, Soerjomataram I et al. Global cancer statistics 2018: GLOBOCAN estimates of incidence and mortality worldwide for 36 cancers in 185 countries. CA Cancer J. Clin. 68(6), 394-424 (2018).

2. Zappa C, Mousa SA. Non-small cell lung cancer: current treatment and future advances. Transl. Lung Cancer Res. 5(3), 288-300 (2016).

3. Gainor JF, Varghese AM, Ou SH et al. ALK rearrangements are mutually exclusive with mutations in EGFR or KRAS: an analysis of 1,683 patients with non-small cell lung cancer. Clin. Cancer Res. 19, 4273-4281 (2013).

4. Wong DW, Leung EL, So KK et al. The EML4-ALK fusion gene is involved in various histologic types of lung cancers from nonsmokers with wild-type EGFR and KRAS. Cancer 115, 1723-1733 (2009).

5. Koivunen JP, Mermel C, Zejnullahu K et al. EML4-ALK fusion gene and efficacy of an ALK kinase inhibitor in lung cancer. Clin. Cancer Res. 14, 4275-4283 (2008).

6. Rodig SJ, Mino-Kenudson M, Dacic S et al. Unique clinicopathologic features characterize ALK-rearranged lung adenocarcinoma in the western population. Clin. Cancer Res. 15(16), 5216-5223 (2009).

7. Solomon BJ, Kim D, Wu Y et al. Final overall survival analysis from a study comparing first-line crizotinib versus chemotherapy in ALK-mutation-positive non-small-cell lung cancer. J. Clin. Oncol. 36(22), 2251-2258 (2018).

8. Planchard D, Popat S, Kerr K et al. Metastatic non-small cell lung cancer: ESMO Clinical Practice Guidelines for diagnosis, treatment and follow-up. Ann Oncol. 29(Suppl. 4), iv192-iv237 (2018).

9. NCCN Clinical Practice Guidelines in Oncology. Non-small cell lung cancer (2019). www.nccn.org/professionals/physician_gls/pdf/nscl.pdf

10. Katayama R, Shaw AT, Mino-Kenudson M et al. Mechanisms of acquired crizotinib resistance in ALK-rearranged lung cancers. Sci. Transl. Med. 4(120), 120ra17 (2012).

11. Costa DB, Shaw AT, Ou SI et al. Clinical experience with crizotinib in patients with advanced ALK-rearranged non-small-cell lung cancer and brain metastases. J. Clin. Oncol. 33(17), 1881-1888 (2015).

12. Camidge DR, Dziadziuszko R, Peters $S$ et al. Updated efficacy and safety data and impact of the EML4-ALK fusion variant on the efficacy of alectinib in untreated ALK-positive advanced non-small-cell lung cancer in the global Phase III ALEX study. J. Thorac. Oncol. 14(7), 1233-1243 (2019).

13. Soria JC, Tan DSW, Chiari R et al. First-line ceritinib versus platinum-based chemotherapy in advanced ALK-rearranged non-small-cell lung cancer (ASCEND-4): a randomised, open-label, Phase III study. Lancet 389(10072), 917-929 (2017). 
14. Shaw AT, Kim TM, Crino L et al. Ceritinib versus chemotherapy in patients with ALK-rearranged non-small-cell lung cancer previously given chemotherapy and crizotinib (ASCEND-5): a randomised, controlled, open-label, phase 3 trial. Lancet Oncol. 18(7), 874-886 (2017).

15. Kim DW, Tiseo M, Ahn MJ et al. Brigatinib in patients with crizotinib refractory anaplastic lymphoma kinase-positive non-small-cell lung cancer: a randomized, multicenter phase II trial. J. Clin. Oncol. 35(22), 2490-2498 (2017).

- Randomized Phase II trial demonstrating substantial whole-body and intracranial responses as well as robust PFS with brigatinib in patients with crizotinib-refractory ALK+ NSCLC.

16. Camidge DR, Kim HR, Ahn MJ et al. Brigatinib versus crizotinib in ALK-positive non-small-cell lung cancer. N. Engl. J. Med. 379(21), 2027-2039 (2018).

- Randomized Phase III trial demonstrating significantly longer PFS with brigatinib compared with crizotinib among patients with $A L K+$ NSCLC who had not previously received ALK TKIs.

17. Camidge DR, Kim DW, Tiseo $\mathrm{M}$ et al. Exploratory analysis of brigatinib activity in patients with anaplastic lymphoma kinase-positive non-small-cell lung cancer and brain metastases in two clinical trials. J. Clin. Oncol. 36(26), 2693-2701 (2018).

- Analysis of Phase I/II and Phase II trials showing brigatinib yields substantial intracranial responses and durable intracranial PFS in ALK+ NSCLC patients treated with crizotinib.

18. Solomon BJ, Besse B, Bauer TM et al. Lorlatinib in patients with ALK-positive non-small-cell lung cancer: results from a global phase 2 study. Lancet Oncol. 19(12), 1654-1667 (2018).

19. Gainor JF, Dardaei L, Yoda $S$ et al. Molecular mechanisms of resistance to first- and second-generation ALK inhibitors in ALK-rearranged lung cancer. Cancer Discov. 6(10), 1118-1133 (2016).

20. Lin JJ, Riely GJ, Shaw AT. Targeting ALK: precision medicine takes on drug resistance. Cancer Discov. 7(2), 137-155 (2017).

21. US FDA Pfizer Inc. Lorbrena (lorlatinib) [package insert] (2020). www.accessdata.fda.gov/drugsatfda_docs/label/2018/210868s000lbl.pdf

22. Reck M, Mok TSK, Nishio M et al. Atezolizumab plus bevacizumab and chemotherapy in non-small-cell lung cancer (IMpower150): key subgroup analyses of patients with EGFR mutations or baseline liver metastases in a randomised, open-label phase 3 trial. Lancet Respir. Med. 7(5), 387-401 (2019).

23. Descourt R, Perol M, Rousseau-Bussac G et al. Brigatinib in pretreated patients with ALK-positive advanced NSCLC. Lung Cancer. 136, 109-114 (2019).

- Evidence of real-world efficacy of brigatinib in heavily pretreated patients with ALK+ NSCLC.

24. Hochmair M, Weinlinger C, Schwab S et al. Treatment of ALK-rearranged non-small-cell lung cancer with brigatinib as second or later lines: real-world observations from a single institution. Anticancer Drugs 30(7), e0787 (2019).

- Evidence of real-world efficacy of brigatinib in heavily pretreated patients with ALK+ NSCLC.

25. Lin HM, Pan X, Hou P et al. Treatment duration of brigatinib in patients enrolled in the international Expanded Access Program (EAP). Ann. Oncology. 30(Suppl. 2), ii38-ii68 (2019).

26. Blumenthall GM, Gong Y, Kehl K et al. Analysis of time-to-treatment discontinuation of targeted therapy, immunotherapy, and chemotherapy in clinical trials of patients with non-small cell lung cancer. Ann. Oncol. 30(5), 830-838 (2019).

27. Lin HM, Pan X, Hou P et al. PCN308 Real-world utilization of brigatinib in patients with non-small cell lung cancer (NSCLC). Value Health 22(Suppl. 2), S115 (2019).

28. DiBonaventura MD, Wong W, Shah-Manek B et al. Real-world usage and clinical outcomes of alectinib among post-crizotinib progression anaplastic lymphoma kinase positive non-small-cell lung cancer patients in the USA. Onco. Targets. Ther. 11, 75-82 (2017).

29. Bendaly E, Dalal AA, Culver K et al. Treatment patterns and early outcomes of ALK-positive non-small cell lung cancer patients receiving ceritinib: a chart review study. Adv. Ther. 34, 1145-1156 (2017).

30. Stinchcombe T, Doebele RC, Wang XF et al. Preliminary results of single arm Phase II trial of brigatinib in patients (pts) with progression disease (PD) after next-generation (NG) anaplastic lymphoma kinase (ALK) tyrosine kinase inhibitors (TKIs) in ALK + non-small cell lung cancer (NSCLC). J. Clin. Oncol. 37(Suppl. 15), 9027 (2019).

31. Novello S, Brustugun OT, Cadranel J et al. Brigatinib in ALK TKI-pretreated ALK+ metastatic non-small cell lung cancer (mNSCLC): the use via expanded access to brigatinib (UVEA-Brig) study. Ann. Oncol. 30(Suppl. 5), mdz260.067 (2019). 
